\title{
Le rock « cosmique » à Berlin-Ouest, bande sonore de la Guerre Froide
}

Der westberliner "Krautrock" als Soundtrack zum Kalten Krieg

"Cosmic" Rock in West Berlin: The soundtrack to the Cold War

\section{Christophe Pirenne}

\section{(2) OpenEdition}

\section{Journals}

Édition électronique

URL : https://journals.openedition.org/ceg/8203

DOI : $10.4000 /$ ceg. 8203

ISSN : 2605-8359

Éditeur

Presses Universitaires de Provence

Édition imprimée

Date de publication : 1 mai 2013

Pagination : 131-145

ISBN : 0751-4239

ISSN : 0751-4239

\section{Référence électronique}

Christophe Pirenne, « Le rock « cosmique » à Berlin-Ouest, bande sonore de la Guerre Froide », Cahiers

d'Études Germaniques [En ligne], 64 | 2013, mis en ligne le 02 janvier 2020, consulté le 02 juin 2021.

URL : http://journals.openedition.org/ceg/8203 ; DOl : https://doi.org/10.4000/ceg.8203 


\title{
Le rock « cosmique " à Berlin-Ouest, bande sonore de la Guerre Froide
}

\author{
Christophe PIRENNE \\ Université de Liège
}

\section{Géographie politique}

Dans l'imaginaire occidental, la séparation physique de la ville de Berlin constitue sans doute l'expression la plus emblématique des tensions et des conflits latents qui opposèrent les blocs de l'Est et de l'Ouest au lendemain de la Seconde Guerre mondiale. Dès la conférence de Potsdam en juillet-août 1945, Berlin est conjointement administrée par les Alliés. La ville est alors divisée en quatre secteurs dévolus respectivement à la France, à la GrandeBretagne, à l'Amérique et à l'URSS.

Les nombreuses tensions qui naissent entre les parties de la ville et surtout l'exode massif de citoyens de l'Est désireux de s'installer à Berlin-Ouest incitent le gouvernement de la RDA à transformer la ligne de démarcation avec Berlin-Ouest en une frontière étanche. La nuit du 12 au 13 août 1961, des barbelés sont posés à tous les points de passage. Deux jours plus tard, des murs de béton commencent à les remplacer. Pendant vingt-huit ans, le Mur divisera la ville, séparant les familles, coupant les quartiers et les rues.

Les inconvénients liés à la situation de Berlin-Ouest, devenue désormais une sorte d'île enclavée, étaient importants et le nombre d'habitants se mit à décrôtre' ${ }^{\prime}$. Chute de la nuptialité dès 1962, de la natalité dès 1964 et taux de mortalité particulièrement élevé. Ces chiffres sont le fruit de l'exode et de flux migratoires particuliers. Au lendemain de la construction du Mur, une partie de la population jeune s'en va et le phénomène n'est pas compensé par

1 En 1939, il y a 2,7 millions d'habitants dans ec qui correspond à Berlin-Ouest, 2 millions au lendemain de la chute du III $^{\mathrm{e}}$ Reich, et malgré une reprise rapide après la guerre, en 1970, il y a moins d'1,9 millions d'habitants. Cela cst dû à deux phénomènes : dès la fin des annécs 50 , les décès enregistrés annucllement à Berlin-Ouest sont plus nombreux que les naissances, unc décroissance naturelle que l'on constate dans toute les villes, mais qui n'apparait souvent que bien plus tard (1968 à Hambourg); il n'y a pas pour Berlin-Ouest de territoire environnant qui fasse office de champ potenticl pour des migrations eentrifuges. Jacques VERON, « Essor et déclin d'une ville : Berlin », Population, 4-5 (1983), p. 866-871. 
l'immigration en provenance de Turquie, de Grèce ou du Portugal. En 1970, $21,4 \%$ des habitants de Berlin-Ouest ont plus de 65 ans, contre $13,2 \%$ dans le reste de la République fédérale d'Allemagne ${ }^{2}$.

Pour toute une bohème allemande cette situation avait néanmoins quelque chose de séduisant. Attirés autant par le statut étrange de la ville que par les innombrables immeubles abandonnés, de nombreux jeunes transforment Berlin-Ouest en refuge de la contre-culture. On y retrouve, comme dans de nombreuses villes occidentales, un large éventail d'activistes politiques, de musiciens diversement compétents et d'apprentis managers qui s'agitent, parfois sous l'influence des drogues les plus diverses, dans des clubs éphémères et des festivals désorganisés. Leurs rencontres, leurs relations vont conduire à la création d'un genre musical neuf et particulièrement foisonnant dans les années 1970, connu sous le nom de rock cosmique. Sa trajectoire, passant d'un cénacle local à un monde global épouse en tous points un modèle théorique développé par Lena et Peterson ${ }^{3}$. Ceux-ci relèvent en effet qu'une bonne partie des genres musicaux passe par une évolution en quatre phases. Des postures esthétiques avant-gardistes se transforment parfois en scène, puis passent par une phase d'industrialisation avant de terminer une sorte de cycle en défendant des postures plutôt conservatrices. Mais le répertoire «cosmique » se caractérise aussi par son irréductibilité aux influences anglo-américaines et par le prestige considérable dont il jouira lors du développement ultérieur de certaines branches majeures des musiques de danse électroniques.

\section{La constitution d'une avant-garde}

Les acteurs qui à Berlin participent à l'élaboration de cette forme de rock sont peu nombreux. En comptant les membres même les plus éphémères de la poignée de groupes qui instaure le genre (Tangerine Dream, Ash Ra Temple, Agitation Free, Kluster), on arrive à une quarantaine de personnes qui peuvent être regroupées en deux générations. Il y a d'abord les initiateurs, nés peu avant ou pendant la Seconde Guerre mondiale. Tous ne sont pas des Berlinois, mais tous partagent la caractéristique d'être très impliqués dans l'art contemporain. C'est le cas de Thomas Kessler, compositeur d'origine suisse qui dirige une Académie de Musique dans le quartier de Wilmersdorf; de Hans Joachim Roedelius né à Berlin-Est et élève de Joseph Beuys; de Dieter Moebius compositeur originaire de Suisse, mais aussi de Conrad Schnitzler et d'Edgar Froese.

Ces artistes opèrent dans les milieux underground où ils font figure de mentors pour une seconde génération de musiciens, plus importante, qui

2 Statistisches Jahrbuch 1982 für die Bundesrepublik Deutschland, Statistisches Bundesamt

3 Jennifer C. LENA, Richard A. PETERSON, «Classification as Culture: Types and Trajectories of Music Genres », American Sociological Rewiew, $73 / 5$ (2008), p. 697-718. 
comprend des enfants de la Guerre Froide, presque tous nés à Berlin entre 1947 et 1953. Ceux-ci sont généralement issus de milieux bourgeois et habitent des quartiers plus favorisés de Berlin-Ouest (Charlottenburg, Wilmersdorf). Ils fréquentent les lycées (Gymnasien) et, parfois grâce à leurs parents, découvrent la musique classique ou le jazz avant le rock. Le cas de Lutz Kramer, membre d'Agitation Free est exemplaire. Il naît à Potsdam en 1952, d'un père acteur puis plus tard producteur de télévision et d'une mère chanteuse d'opéra et professeur de chant. Il passe son enfance dans l'arrondissement de Charlottenburg où il commence à étudier le piano dès l'âge de sept ans. Il entre ensuite dans un Gymnasium où il fait la rencontre de certains des futurs membres d'Agitation Free.

Comme toute avant-garde, ces artistes partagent un dégoût pour la musique de leur temps. Leur but, du moins à l'origine, est de rejeter les principes d'écriture classique (rythme, forme, harmonie, mélodie) et de transposer l'expérience des arts visuels en musique. Le résultat de ces expérimentations consistera souvent en longues pièces instrumentales réalisées avec des instruments électriques dont ils jouent d'une manière non conventionnelle. À partir de 1973, une part de leurs expérimentations s'opérera exclusivement avec des synthétiseurs.

\section{La constitution d'une scène}

La communauté alternative qui se développe à Berlin-Ouest à la fin des années 1960 va s'agglomérer dans quelques clubs underground. Le Zodiak Club, créé en 1968 par Conrad «Conny » Schnitzler et Hans Joachim Roedelius en est la figure de proue. Défini par ses fondateurs comme un experimental art lab, il est installé dans la cave d'un célèbre théâtre, la Schaubühne, alors située « am Halleschen Ufer, 32 » dans Kreuzberg. Les concerts ne peuvent y commencer qu'après les représentations. C'est dans ce club que les nouveaux groupes apprennent à se connaître. Kluster, Tangerine Dream, Eruption, Curly Curve et Agitation Free y jouent en alternance avec des musiciens plus radicaux dont Conrad Schnitzler, Dieter Moebius et HansJoachim Roedelius. Le club s'affirme donc comme un point de rencontre pour les musiciens de rock et d'avant-garde. Ainsi, Agitation Free y est introduit par Volker Cornelius, un étudiant en architecture habitant dans Charlottenburg. Dès 1968, le groupe fait presque office de groupe en résidence, puisqu'on peut l'y entendre parfois plusieurs fois par semaine.

Lorsque le Zodiak Club ferme à cause des problèmes de drogue, la plupart des groupes se relogent au Beautiful Balloon. Celui-ci est installé dans les locaux du Lichtspieltheater sur la Lehniner Platz ${ }^{4}$. Le concert d'ouverture est assuré par le " power trio » anglais The Nice, et de manière générale, ce sera l'un des principaux lieux d'accueil des groupes étrangers en tournée. Le

4 C'est la Schaubühne qui y emménagera par la suite. 
problème de l'endroit ce sont les prix d'accès puisque les concerts coûtent de 12 à 15 DM. Le lieu est néanmoins important puisque c'est là que Folke Hanfeld introduira les premiers spectacles multimédias.

À côté de ces clubs essentiels, on trouve le Sun, première discothèque underground berlinoise, au carrefour du Kurfürstendamm et de la JoachimFriedrich-Straße, dans Wilmersdorf; le Sound, une grande discothèque connue à ses débuts sous le nom de Centrum 2000. Agitation Free y joue dès 1968 dans un «beat music contest ». Le lieu allait devenir une sorte de Mecque des drogues dures et acquérir une notoriété internationale avec la publication de l'autobiographie de Christiane Felscherinow, Wir Kinder vom Bahnhof Zoo (1978) ; enfin, le Quartier Latin au numéro 26 de la Potsdamer Straße, proposera à partir de 1972 des « Total Music Meetings ».

À côté des lieux officiellement consacrés à la musique, la Mensa, le restaurant universitaire de l'Université Technique de Berlin (Technische Universität, TU) située sur la Hardenbergstraße, fut aussi un lieu de ralliement privilégié. Ouverte aux concerts ou aux lectures elle se révèle être un point d'accrétion d'autant plus apprécié qu'il n'y a pas de frais de location. Tous les groupes locaux débutants s'y produisent de même que de nombreuses formations d'Allemagne de l'Ouest alors à l'aube de leur carrière (Guru Guru, Can, Amon Düül). À proximité de l'Université, quelques salles voient aussi le jour dont le Quasimodo, situé dans la cave du Delphi Filmpalast, à l'angle de la Kantstraße et de la Fasanenstraße. Dès le début des années 1970 des étudiants viennent y écouter du dixieland, puis y seront organisés des concerts de free jazz, avant que toute une série de groupes locaux dont Tangerine Dream et Curly Curve ne s'y produisent.

Parmi les lieux fondateurs, les squats qui fleurissent à Berlin-Ouest à la fin des années 1960, jouent un rôle tout à fait central. La Kommune 1, la première communauté résidentielle urbaine, est fondée en $1967^{5}$. Ses membres s'installent entre autres dans une ancienne usine de la Stephanstraße, située dans le quartier de Moabit. On y découvre le mélange habituel d'activisme politique, de musique underground, de participation à des happenings ou à des teach in et de rejet des valeurs bourgeoises. Parmi ses membres les plus emblématiques on compte Rainer Langhans, lequel fut un bref moment roadie d'Agitation Free, Lutz «Ludwig » Kramer, guitariste d'Agitation Free, de même que Karl-Heinz Hausmann, futur claviériste d'Amon Düül II à partir de 1971. Cela ne signifie pas pour autant que les musiciens reprennent à leur compte les idées radicales développées par certains membres de Kommune 1. Au contraire, hormis Ludwig Kramer cette scène musicale berlinoise sera fort peu impliquée dans les combats politiques de l'époque.

5 La littérature consacrée à la Kommune I est abondante. Ulrich ENZENSBERGER, Die Jahre der Kommune I. Berlin 1967-1969, Köln, Kiepenheuer \& Witsch, 2004. On peut également évoquer l'ouvrage Christa RITTER, Rainer LANGHANS, Herz der Revolte. Die Kommune I von 1967 bis 1969, Hannibal Verlag, 2005. 
Enfin, le dernier lieu de rassemblement de cette communauté d'artistes est le Beat Studio de Thomas Kessler situé sur la Pfalzburger Straße et rattaché à l'école de musique de Wilmersdorf. À partir de 1968, il y installe de petits locaux de répétition, dans lesquels, au cours des années suivantes Nosehead, Agitation Free, Tangerine Dream, Ash Ra Tempel et quelques groupes de blues ou de jazz rock vont s'exercer. Là où l'action de Kessler semble avoir été décisive, c'est qu'en parallèle, il propose à ses hôtes des cours de théorie musicale et les introduit dans le monde de l'électronique musicale. En fréquentant le Beat Studio, les musiciens reçoivent non seulement une formation, mais ils ont aussi l'occasion de se rencontrer, créant des liens qui conduisent à la formation ou à la reconfiguration de certains groupes. Agitation Free est l'un des premiers à fréquenter le Beat Studio. En 1971, ses membres incitent Michael Hoenig, lequel est inscrit dans la même école secondaire que Michael Gunther, à assister à leurs répétitions et à entrer dans le groupe d'improvisation de Thomas Kessler. C'est là qu'il apprend à travailler avec des bandes préparées. Hoenig intégrera finalement Agitation Free et dans cette nouvelle configuration, le groupe jouera à la Mensa de la TU où, pour la première fois, le public pourra entendre des sons électroniques produits par un générateur de fréquences.

Plusieurs musiciens témoignent de l'importance de Kessler :

\begin{abstract}
A music director named Thomas Kessler gave them [Agitation Free] a room of their own for practicing. He wanted to take care of us because we were only fifteen or sixteen at the time. So he became a sort of a mentor. He taught us about electronic music and avant-garde music. They installed reel-to-reel tape machines and microphones, and Kessler showed us how to make "musique concrète » with them. We tried to bled these things into our experimental rock music. We couldn't rehearse more than twice a week there anyway. Since the equipment was there and the place was made soundproof, we had a nice kind of basement room. We decided that this place should also be open for other interested people. So other people came who have since become known as the Berlin electronic scene. ${ }^{6}$
\end{abstract}

À ces propos de Christoph Franke répondent, entre septembre et décembre 1971, ceux de Michael Gunther qui écrit :

Thomas Kessler began furiously to coach us: ear-training, harmony, music theory, rhythm. He tried all kinds of things, conducted experiments, and afforded us a considerable insight into the world of classical music.

C'est également Thomas Kessler qui, en introduisant Peter Michael Hamel en 1971, incita Agitation Free à enregistrer. L'implication du groupe dans le Beat Studio eut une étonnante répercussion. Entre janvier et mars 1972, Agitation Free allait inviter des professeurs de musique et leurs élèves dans le

6 Christoph FRANKE : http://www.sonicimages.com (consulté en novembre 2012, comme l'ensemble des références internet signalées dans le présent article).

7 Michael GUNTHER : http://www.agitation-free.de/wechsele.html. 
Beat Studio afin de les initier à : " l'avant-garde, le rock et la musique électronique $»^{8}$.

Agitation Free est sans doute le groupe le plus central de la scène berlinoise dans la mesure où son existence un peu chaotique à ses débuts et les multiples changements de personnel en ont fait une sorte de vivier pour la plupart des autres formations berlinoises. Christopher Franke et Michael Hoenig passeront chez Tangerine Dream, le second formant ensuite Ash Ra Tempel ; Manuel Göttsching, Hartmut Enke, Klaus Freudigmann, Conrad Schnitzler, Thomas Keyserling et Michael Gunther créeront pour leur part un groupe d'improvisation de musique «sérieuse » qu'ils appellent Eruption. Ce n'est qu'en 1971 qu'Agitation Free devient un groupe plus ou moins stable. Dès l'origine, il fait fonction de groupe en résidence au Zodiak Club, et bien plus que d'autres formations berlinoises, il tisse des liens avec des groupes politiques de gauche. Les prestations d'Agitation Free se caractérisent par leur côté happening, mêlant effets optiques avant-gardistes et longues pièces musicales largement improvisées, faisant alterner des passages tout à fait rock avec des collages sonores inspirés de la musique électronique. Dès l'origine, Folke Hanfeld, le frère du bassiste des Sentries (première incarnation d'Agitation Free) travaille dans l'orbite du groupe et s'occupe des jeux de lumière. Dès la fin de l'année 1967, il utilise des wet gel ${ }^{9}$, des diapositives, et des films de $8 \mathrm{~mm}$ qu'il tournait lui même ${ }^{10}$. À cet aspect multimédia s'ajoute aussi un volet participatif. Comme l'écrit Lutz Ludwig Kramer, guitariste et chanteur du groupe, tous tentaient de remettre en cause et faire bouger les frontières entre le public et le groupe. Les instruments pouvaient être distribués aux spectateurs et tout se combinait de manière organique ${ }^{11}$. En ce sens, le développement des pratiques musicales berlinoises ne diffère pas beaucoup de ce qui se fait chez leurs homologues anglais, d'autant que la base de leur répertoire est constituée de reprises. Ils s'en éloignent peu à peu en insérant des passages improvisés qui finissent par devenir l'essentiel. Comme le signale Michael Gunther : « C'est comme cela que nous nous amusions : en allongeant les reprises et par dessus tout en improvisant de nouveaux passages. C'était très différent de ce que nous entendions sur la plupart des disques de l'époque $»^{12}$.

Ash Ra Temple, autre groupe quintessentiel de cette scène berlinoise, est formé en juin 1969 sous le nom de Steeple Chase Bluesband. La formation originale, constituée par Manuel Göttsching (guitare), Hartmut Enke (basse) et Wulf Arp (batterie), ainsi que par Volker Zibell (harmoniciste), qui rejoint le groupe en août 1969, joue un blues rock très fortement influencé par John

8 Michael GUNTHER : http://www.agitation-free.de/cairod.html

9 Une gélatine placée entre deux verres, eux-mêmes disposés devant une source lumineuse Sous l'action de la chaleur dégagée par le spot, le gel se meut et produit des effets visuels particulièrement appréciés par les artistes psychédéliques.

10 Pour la biographie du groupe voir : http:/www.agitation-free.de.

11 Cité dans http://www.lutzludwig.de/indexe.html.

$12 \mathrm{http}: /$ www.agitation-free.de/anfange.html. 
Mayall. Le répertoire d'Ash Ra Tempel se met peu à peu en place lorsque Klaus Schulze quitte Tangerine Dream et s'associe à Manuel Gottsching et Hartmut Enke, à une époque où tous trois étaient impliqués dans Eruption (cf. supra). Ils commencent à jouer ensemble en août 1970, et se créent une certaine renommée par leurs longues improvisations. Après avoir enregistré quelques démos, ils se rendent à Hambourg pour enregistrer leur premier album éponyme, publié par Ohr en 1971. Ce sera le seul album enregistré avec Klaus Schulze qui quitte alors la formation pour se lancer dans sa carrière solo.

La scène berlinoise voit aussi éclore le groupe déjà mentionné Tangerine Dream, un quintette formé en 1967 par Edgar Froese avec Kurt Herkenberg (basse), Volker Hombach (violon, flûte, chant), Lanse Hapshash (batterie) et Charlie Prince (chant) comme membre occasionnel. Les premiers concerts de Tangerine Dream ont lieu dès janvier 1967, et le groupe s'impose comme le premier de la scène berlinoise à tenter de jouer en dehors de la capitale dans des concerts où s'affirment ses liens avec les arts plastiques contemporains. On les entend par exemple à la Documenta IV de Kassel où ils animent le concert d'ouverture, à Port Lligat en Espagne où ils jouent pour l'inauguration de la «Statue du Christ » de Salvator Dali. À Berlin, où on les entend au Zodiak Club, à la Mensa de la TU et également lors du happening organisé au Forum Theater. Après ces concerts, Froese dissout la première incarnation de Tangerine Dream et en reforme une seconde avec Klaus Schulze et Conrad Schnitzler. Froese avait rencontré Schnitzler à la fin de l'année 1969 lors du vernissage d'une exposition consacrée à Joseph Beuys. Schnitzler, lui-même un élève de Beuys, accepte de participer, comme violoncelliste aux sessions d'enregistrement du premier album du groupe, Electronic Meditation, en octobre 1969, dans un loft de Kreuzberg. Ce disque aussi sera publié par le label Ohr.

En 1971, Klaus Schulze se lance dans une carrière solo et Tangerine Dream connait alors sa troisième incarnation, toujours centrée autour d'Edgar Froese qui s'adjoint cette fois les services de Christoph Franke (d'abord à la batterie puis au synthé) et de Steve Schroyders (orgue), tous deux issus d'Agitation Free. Les deux premiers se rencontrent à l'école de musique de Thomas Kessler ou au cours d'une session d'enregistrement durant laquelle Edgar Froese devait co-produire une version de The Boxer de Simon et Garfunkel pour un artiste allemand ${ }^{13}$.

Lorsque j'ai rencontré Edgar, mon groupe, Agitation Free était en train de se dissoudre. Il était lui-même dans une période de transition. Tangerine Dream s'était séparé parce que Klaus Schulze et Conrad Schnitzler venaient juste de quitter le groupe. Dès le début, Edgar et moi nous entendîmes à merveille. Nous étions très désireux de travailler ensemble parce que nous voulions tous deux nous éloigner de la musique écrite. Nous voulions faire de longues chansons et improviser. Tous les horizons s'ouvraient à nous - non seulement ceux de la musique classique et de la musique électronique, mais aussi ceux des musique

13 La relation de cette rencontre se trouve sur le site http://voices-in-the-net.de. 
Indiennes et Africaines. C'était une sorte de guide. Lorsque j'ai décidé de jouer avec lui, je me suis dépouillé de mon éducation, et c'est à partir de ce moment que j'ai travaillé comme musicien professionnel. Comme je devais avoir 17 ans, ce sont mes parents qui durent signer mon premier contrat discographique. Edgar avait neuf ans de plus que moi. ${ }^{14}$

L'existence du trio sera de très brève durée. Conrad Schnitzler s'en va rapidement pour travailler avec Kluster, puis avec Eruption tout en travaillant pour le Zodiak Club. Il est remplacé, au début de 1971 par Steve Schroyder. Ce dernier n'est pas Berlinois. Il est arrivé à Berlin en 1968 pour y effectuer des études de théâtre, mais c'est aussi un musicien averti puisqu'il a suivi des cours de piano pendant près de dix ans. En sa compagnie, Tangerine Dream enregistre l'album Alpha Centauri, qui comme son prédécesseur repose d'une part sur la volonté de transférer l'expérience des arts visuels en musique, et d'autre part sur le désir de se dépouiller des diktats de l'harmonie classique. Pour ce disque, Christoph Franke avait découvert à Londres son premier synthé électronique. Il s'agissait d'un petit EMS «suitcase » qui devint un élément sonique central dans l'album. Avec Schroyder, ils développent aussi un son d'orgue original qui contribuera à créer, à partir de ce moment leur image « spatiale».

La constitution de la scène berlinoise ne se limite pas à des lieux et à des groupes. Elle repose aussi sur les initiatives d'entrepreneurs issus de ses rangs. Pour les groupes Berlinois, Rolf-Ulrich Kaiser fut cet homme providentiel. Même s'il n'avait pas de liens directs avec Berlin il partageait les mêmes goûts et les mêmes modes de vie que les groupes berlinois. Il avait été l'un des initiateurs des Internationale Essener Songtage (25-29 septembre 1968), un festival qui avait servi de plateforme à de nombreux groupes allemands «progressistes » dont Tangerine Dream puis, dans la foulée, il avait publié quelques ouvrages servant de véritables manifestes aux musiques underground allemandes. En compagnie de Peter Meisel, directeur de la maison d'édition musicale Hansa, il avait fondé la maison de disques Ohr. En quelques mois, celle-ci devint le refuge d'une bonne partie des groupes "progressistes " berlinois, publiant les premiers disques de Tangerine Dream : Electronic Meditation (1970), Alpha Centauri (1971), Zeit (1972) et Atem (1973); d'Ash Ra Tempel : Ash Ra Tempel (1971), Schwingungen (1972) et Join Inn (1973) ainsi que de Klaus Schulze : Irrlicht (1972). Sur les quelque trente-trois LPs publiés par Ohr entre 1969 et 1973, les Berlinois ne représentent cependant qu'une portion congrue de sorte que l'idée d'un son «berlinois » s'amenuisa au profit d'un son « allemand».

La terminologie utilisée à l'époque pour décrire ces musiques témoigne de la difficulté de lier un son à un lieu. À Berlin comme dans les autres pays occidentaux, les groupes musicalement les plus aventureux sont d'abord regroupés sous les appellations « rock progressif», «pop progressif » ou «underground music ». Dans un élan tout aussi convenu, les promoteurs de

14 Christopher FRANKE : http://www.sonicimages.com. 
cette scène réfutent initialement toute tentative d'appellation : "Ohr-Platten brauchen keine Modewörter, kein 'Underground', kein 'Blues', kein 'progressiv'. Mach das Ohr auf? Deutschlands eigene Pop-Musik: OhrKlänge ${ }^{15}$ ("Les disques Ohr n'ont pas besoin de mots à la mode, d' 'underground', de 'blues', de 'progressiste'. Ouvre l'oreille [das $\mathrm{Ohr}$ ] ! L'Allemagne possède sa propre musique pop : le son $\mathrm{Ohr} »)$. Quelques mois plus tard cependant, la musique produite par les groupes berlinois se voit désignée par une appellation singulière. Celle de Elektronischer Rock ou de Elektronischer Free Rock. Elle est d'abord employée pour décrire Electronic Meditation (1970) de Tangerine Dream - lequel ne comporte paradoxalement aucun instrument électronique16 - et on la trouve aussi dans la description des premières prestations de Popol Vuh ${ }^{17}$. En dehors de ces cas isolés, l'appellation ne s'impose pas, en tout cas pas pour qualifier une branche du rock pratiquée par des groupes allemands.

Les termes Kosmische Musik n'apparaissent qu'à l'automne 1972. Ils étaient pourtant dans l'air du temps depuis plusieurs années. Dès 1968, Zodiac, un groupe anglais justement oublié, semble avoir été le premier à utiliser le terme « cosmique » dans le domaine de la musique pop en publiant chez Elektra un album intitulé Cosmic sound. Mais au-delà de cette apparition anecdotique, c'est l'émergence d'une approche un peu mystique de la musique qui semble mettre le terme à la mode. Dans sa fameuse Lettre ouverte à la jeune génération, Karlheinz Stockhausen écrivait ainsi : « Musik sollte ein [...] Strom von überbewußter kosmischer Elektrizität sein ${ }^{18}(« \mathrm{La}$ musique devrait être un courant d'électricité cosmique et super-consciente »). C'est Rolf-Ulrich Kaiser qui semble être l'initiateur de l'association des mots « cosmique » et «rock» ${ }^{19}$. C'est en tout cas le titre qu'il choisit pour présenter la double compilation éponyme ${ }^{20}$ éditée en même temps que neuf disques de Kosmische Musik parmi lesquels on touve Irrlicht Quadrophonische Symphonie für Orchester und E-Maschinen de Klaus Schulze. Si ce terme connaît rapidement une fortune importante, c'est en partie parce que Kaiser l'utilise massivement comme slogan publicitaire. La presse commence alors à s'en emparer lorsqu'elle signale par exemple que «[Der] Komponist Klaus Schulze wird Ende Oktober zusammen mit dem Thema 'Kosmische Musik' in allen Rundfunkstationen vorgestellt » ${ }^{21}$ ( Fin octobre, le compositeur Klaus Schulze et la 'musique cosmique' sont présentés sur toutes les stations radio $»)$, mais l'usage ne se répand que

15 [s.n.], Musikmarkt, 6/2/70, p. 1 .

$16 \mathrm{PH}$, « Tangerine Dream : Electronic Meditation », Sounds, 20/70, [n.p.].

17 Sounds, 23/70.

18 Karlheinz STOCKHAUSEN, « Ein offener Brief an die junge Generation », Sounds, $17 / 70$, [n. p.]

19 Siegfried NIEDERGESÄß, "Wir fördern den Trend zum deutschen Text», Musikmarkt, $13 / 72$, p. 42.

20 Le sampler « Kosmische Musik » sort finalement au début de l'année 1973. [s.n.], «Ohr:,Floh‘ als Mitschnitt ». Musikmarkt, 2/73, p. 55.

21 [s.n.], «Ohr : Neue Birth Control », Musikmarkt, 20/72, p. 64. 
lentement, ce dont témoigne par exemple l'utilisation simultanée de terminologies différentes pour expliquer le succès inattendu de $I r r l i c h t^{22}$.

La Cosmic Music fait son entrée dans un lexique consacré au rock en 1973. L'auteur de la notice la présente comme une des catégories de la musique rock des plus vagues et des plus versatiles :

\begin{abstract}
Zum erstenmal tauchte das Attribut bei den Byrds mit Eight Miles High auf, einem Son über Science Fiction, Weltraumfahrt, Zukunft. Oft gebraucht wird die Bezeichnung für Gruppen wie Pink Floyd, King Crimson, Amon Düül II, Curved Air, Genesis, Jimi Hendrix, Zodiac Cosmic Sound, Jefferson Starship, Grateful Dead, Tangerine Dream, also für Musiker, die auf irgendeinem Weg (sei es im Text, in Melodie, Rhythmik, im Klangbild) versuchen, ,Überirdisches in Töne zu fassen oder mit Klängen zu vermitteln. Dazu zählen natürlich auch die Elektroniker, die auf technischem Weg neue Musikformen versuchen: John Cale, Terry Riley, Popol Vuh, Can, Cluster, Kraftwerk, Eberhard Schoener, Ash Ra Tempel, Klaus Schulze, Neu. ${ }^{23}$
\end{abstract}

Rolf-Ulrich Kaiser se servit d'une partie de ce terme pour baptiser sa collection Kosmische Kuriere, contribuant de la sorte à une extension de l'usage du vocable Kosmische Musik à tous les répertoires dominés par les instruments électroniques.

\title{
La constitution d'un genre mainstream
}

Lorsque les groupes de Berlin, et de manière plus large les groupes allemands « cosmiques », "progressistes » ou " électroniques » révèlent leurs potentialités commerciales, le statut de cette scène jusque lors très locale prend une nouvelle ampleur et d'autres maisons de disques s'intéressent à ce filon potentiel.

En février 1972, Metronome, conforté par le succès de Ohr, lance son propre label de musique pop allemande : Brain. Deutsche Grammophon n'hésite pas non plus à impliquer son nom prestigieux dans la distribution du label Kuckuck ${ }^{24}$, puis elle fait un pas supplémentaire à la fin de l'année 1972, lorsqu'elle fonde son propre label progressif Zebra ${ }^{25}$; le premier juin 1971, la firme Miller International lance le label de «progressive-pop » Plus ${ }^{26}$; RCA se joint au mouvement en créant le label à vocation internationale Neon;

22 Les raisons évoquées pour expliquer le succès de Irrlicht témoignent bien de l'ambivalence terminologique : «1. Erste quadrophonische, progressive Produktion, 2. Erste progressive deutsche Pop-Platte mit Symphonieorchester, 3. Ein weiteres erfolgreiches Album der ,Kosmischen Musik'»». [s.n.], Musikmarkt, 22/72, p. 62-64.

23 Ingeborg SCHOBER, Rock-Lexikon, 1973, [n.p.].

24 Hans-Ulrich PROST, "Progressive Musik: Was bedeutet sie ? Wer macht sie ? Wer kauft sie? », Musikmarkt, 8/71, p. 40.

25 [s.n.], « Auf ,Zebra“ kommt man progressiv », Musikmarkt, 22/72, p. 4

26 [s.n.], «,Plus' : Neues Label für Millers Progressiv-Pop », Musikmarkt, 13/71, p. 22. 
BASF enfin distribue Pilz $z^{27}$ un label créé par le tandem Peter Meisel et RolfUlrich Kaiser à partir de novembre $1971^{28}$.

Cette multiplication correspond à un réel succès commercial et, en corollaire, à une multiplication des sorties. En septembre 1970, Haus Liberty/UA lance en Allemagne une collection de douze albums de « progressive Pop-Musik»29. Ariola fait de même et offre, à partir de mai 1971, pas moins de 30 LPs destinés à « un public d'acheteurs progressistes $»^{30}$. « Keine Zweifel, Die Progressiv-Welle überschwemmt nun auch die Bundesrepublik. Nie zuvor wurden so viele Platten mit aktueller Musik abgesetzt wie in diesen Wochen. Und selbst Firmen, die sie bisher auf diesem Gebiet zurückhielten, steigen nun voll ein ${ }^{31}$ ( ( Pas de doutes, la vague progressiste déferle maintenant aussi sur la République Fédérale. Jamais autant de disques de musique actuelle ne furent écoulés que ces dernières semaines. Et même les firmes qui avaient l'habitude de se tenir à l'écart dans ce domaine s'y impliquent désormais totalement »). Signe des temps, ces répertoires progressistes recueillent tant de suffrages qu'ils sont l'objet de querelles de distribution féroces. En 1971, Bellaphon achète le label Bacillus jusque là distribué par Phonogram, afin de disposer d'un « deutscher Progressiv-Katalog $»^{32}$. Quelques mois plus tôt, Phonogram qui distribuait le label «Island» avait été dépouillé de ce label porteur - il constituait à l'époque $10 \%$ de ses recettes - au profit d'Ariola ${ }^{33}$.

Pour la promotion et la diffusion, toutes fonctionnent de la même manière. Les nouveautés sont généralement publiées par groupe de quatre ou cinq disques, et diffusées pas le biais de concerts ou de tournées de promotion. Ainsi, en 1971, la présentation de la troisième série de productions d'Ohr (Birth Control, Tangerine Dream, Witthüser \& Westrupp et Ash Ra Temple) est suivie des «Berliner Rock-Tage ", concerts de présentation des groupes berlinois sous contrat chez $\mathrm{Ohr}^{34}$. Au même moment, Liberty organise sous le titre de « German Progressive Pop Experience », une série de concerts avec «ses» Allemands : Amon Düül II, Can et Krokodil ${ }^{35}$. Plus présente ses groupes (Tommorow's Gift, Ikarus et Wind) lors d'un concert d'intronisation. United Artists fait de même le 24 novembre 1971 dans le Grugahalle de

27 [s.n.], « Neues Progressiv-Label, ,Pilz‘ ab sofort im BASF-Vertrieb », Musikmarkt, $21 / 71$, p. 6 .

28 Siegfried NIEDERGESÄß, «Wir fördern den Trend zum deutschen Text », Musikmarkt, 13/72, p. 42.

29 « Electric Rock - Idee $2000 »$, Musik Information, 9/70, p. 52-53.

30 Hans-Ulrich PROST, «Ein neues Schiff auf aktueller Welle », Musikmarkt, 10/71, p. 11.

31 «"Think-Pop Progress 71": das große Frühjahrsgeschäft », Musik Information, 5/71, p. 6 .

32 [s.n.], « Bellaphon kaufte Bacillus als Label fürs Progressive », Musikmarkt, 21/71, p. 6.

33 [s.n.], «Von Phonogram zu Ariola : Progressiv-Label Island », Musikmarkt, 10/71, p. 10.

34 [s.n.], « Ausland zahlte 100000 DM für die ,Birth Control` », Musikmarkt, 10/71, p. 10.

35 [s.n.], « Zur Image-Verbesserung : Libertys Progressiv-Gruppen auf Tournee », Musikmarkt, 10/71, p. 10 . 
Essen. Pour cinq marks, les spectateurs peuvent assister à un concert de promotion intitulé " United Artists presents United Artists » au cours duquel se produisent, entre autres, Amon Düül II, Can et Embryo ${ }^{36}$. Le succès de l'expérience - le concert est complet - incitera les responsables d'UA à réitérer l'expérience dans d'autres villes dont Berlin ${ }^{37}$. Pilz ne procède pas très différemment lorsqu'il s'agit de lancer sa collection. BASF convie de son côté vingt-cinq journalistes à suivre quelques-uns de ses groupes pendant trois jours au cours d'une mini-tournée en Allemagne. Wallenstein, Hölderlin, Emtidi et Witthüser \& Westrupp donnent des concerts, se prêtent au jeu des interviews et font découvrir aux journalistes l'envers du décor, et les difficultés auxquelles sont confrontés les groupes de la « nouvelle musique pop et rock allemande $"{ }^{38}$.

À la fin de l'année 1972, lorsque Rolf-Ulrich Kaiser tente de présenter le rock cosmique comme une des formes de rock progressif, il use aussi de la même stratégie. La compilation Kosmische Musik ainsi que les disques d'Annexus Quam, d'Ash Ra Tempel, de Mythos et de Tangerine Dream sont proposés dans une offre combinée au public, tandis que la promotion est assurée conjointement par Klaus Schulze et Rolf-Ulrich Kaiser qui effectuent une sorte de tournée d'interviews dans les principales stations de radio ${ }^{39}$.

\section{La constitution d'une tradition}

Si la multiplication des firmes et des collections témoigne de l'implantation du rock underground sur le sol allemand, son internationalisation sera précipitée à la suite de l'anéantissement de l'empire de Rolf-Ulrich Kaiser. En 1973, ce dernier avait réuni une sorte de «all-star group » en studio. Plusieurs musiciens d'Ash Ra Temple et de Wallenstein ainsi que Klaus Schulze s'étaient réunis au cours de plusieurs sessions réparties de février à mai pour improviser et esquisser des compositions, le plus souvent sous l'emprise du LSD. Le projet et le groupe, que Kaiser avait baptisé Cosmic Jokers, se termina de manière peu aimable lorsque les musiciens réalisèrent que Kaiser avait publié, sans leur autorisation et sans leur verser de droits, trois albums d'extraits de ces sessions.

Les péripéties qui s'ensuivent imposent à tous les groupes attachés aux labels de Kaiser (Ohr, Pilz, Kosmischen Kuriere) de trouver d'autres éditeurs. Certains d'entre eux se tournent vers la firme Virgin, un nouveau label indépendant anglais fondé par Richard Branson et Nik Powell en 1972. Tangerine Dream, Faust et, en distribution seulement, Can, soit trois des

36 [s.n.], « Mit fünf Mark sind Fans dabei », Musikmarkt, 22/71, p. 66-67.

37 [s.n.], « Liberty-Experiment geglückt », Musikmarkt, 24/71, p. 26.

38 Siegfried NIEDERGESÄß, « Mit Pop von Pilz auf Promotion-Pfaden », Musikmarkt, $21 / 72$, p. $22-23$

39 [s.n.], « Ohr: Klaus Schulze « gewinnt»», Musikmarkt, 22/72, p. 62 \& 64. [s.n.], «Ohr : Liver-Report der Floh Tournee », Musikmarkt, 24/72, p. 52. 
figures majeures du rock allemand se retrouvent ainsi aux côtés de Mike Oldfield, Robert Wyatt, Gong ou Hatfield and The North dans le catalogue de la firme anglaise indépendante la plus importante de la première moitié de la décennie.

Tangerine Dream sera le plus grand bénéficiaire de cette association. Leur arrivée chez Virgin coïncide avec la parution d'une suite de classiques du rock cosmique : Phaedra (1974), Rubycon (1975), Ricochet (1975) et Stratosfear (1976) ; le troisième de ces disques devenant même numéro un en Angleterre. Leur musique est désormais très différente de celle de leurs premiers albums. Les synthétiseurs restent les instruments centraux du groupe, mais l'expérimentation a disparu au profit d'un retour vers des mélodies très mnémoniques et des sons percussifs qui font entrer le groupe dans l'ère de la musique binaire.

Cette entrée de Tangerine Dream dans le giron de Virgin marque de manière symbolique l'évolution des musiciens berlinois dans la quatrième phase du schéma d'évolution des genres tel qu'il est décrit par Lena et Peterson. Désormais l'innovation diminue au profit de la préservation et de la transmission d'un héritage musical. Les techniques d'écriture se figent, le discours des amateurs se focalise sur la construction d'une mythistoire berlinoise avec une argumentation faisant appel à l'origine géographique, à des albums référentiels et à des artistes iconiques. Cette mondialisation conduit enfin à un nouveau renouvellement de la terminologie. Les répertoires allemands qui s'imposent en Angleterre seront désormais baptisés par la presse anglaise du nom peu flatteur de Krautrock, de «rock choucroute » qu'on peut aussi traduire par « rock des Fritz ou Fridolins » (cf. sens péjoratif de « the krauts » en anglais).

\section{Conclusions}

Le passage du local au global que connaît la scène berlinoise, n'explique pas l'essentiel. Pourquoi, voit-on naître à Berlin une musique aussi différente de ce qui se fait à Londres, à Canterbury ou sur la côte Ouest des ÉtatsUnis alors qu'on y trouve un mélange assez commun de théâtre, d'arts plastiques, de musique contemporaine, de rock, de drogue, de contestation?

Outre le génie propre de certains des membres de cette scène, quelques spécificités contextuelles permettent d'avancer des bribes de réponses. La scène rock berlinoise de la fin des années 1960 a ceci de particulier qu'elle se construit sans musiciens issus de sa banlieue. Alors qu'à Londres par exemple les courants musicaux les plus progressistes sont le fruit d'adolescents de la classe moyenne issus de banlieues aisées, à Berlin cette banlieue n'existe $\operatorname{pas}^{40}$. Il n'y a pas d'arrière-pays. Le son de la ville est donc exclusivement

40 Voir Christophe PIRENNE, Le rock progressif anglais : 1967-1977, Paris, Honoré Champion, 2005, p. 229-232. 
façonné par des citadins ou par ceux qui proviennent de régions plus lointaines.

La seconde spécificité de la scène berlinoise, tout aussi liée aux contingences socio-politiques, tient à la nature de la musique. D'emblée, les journalistes et les amateurs qui s'intéressent aux longues plages méditatives d'Ash Ra Temple ou de Tangerine Dream y décèlent une forme de mimesis. Les sonorités du genre semblaient avoir la capacité de permettre aux auditeurs d'imaginer des lieux, de suggérer l'espace et surtout, dans une ville confinée, de s'en évader : " Dans une ville barrée, écrit l'un d'eux, la musique ne put se développer horizontalement, le rock cosmique est une musique verticale, qui se développe de bas en haut, s'épanouissant vers l'espace ».

À ces aspects peut s'ajouter une troisième spécificité plus proprement liée à l'histoire récente de l'Allemagne. Il semble en effet possible de lire la musique « cosmique » comme l'incarnation des ambiguïtés véhiculées par la réappropriation de l'histoire allemande. Au moment où les musiciens de rock "progressif» de tous les pays occidentaux commencent à se réinscrire dans la tradition musicale occidentale en se réclamant de grands compositeurs du passé ; au moment ou le mouvement général des musique populaires passe du " Roll Over Beethoven » de Chuck Berry au "Switch on Bach » de Walter Carlos, les Allemands semblent plus réservés à l'idée de se réclamer de leur passé national. C'est d'autant plus difficile que les périodes historiques dont se réclament les musiciens anglais sont justement les plus délicates puisqu'ils font massivement référence aux romantiques et aux musiciens " nationalistes" actifs à la fin du XIX ${ }^{\mathfrak{e}}$ siècle et au début du $\mathrm{XX}^{\mathrm{e}}$ siècle. Le choix des artistes berlinois consistera à se réclamer des périodes les plus récentes de leur histoire musicale, soit des œuvres composées durant l'immédiat après-guerre. Le choix de leurs figures tutélaires, de leurs instruments, de leurs références plastiques est à cet égard sans équivoque et très éloigné de pratiques anglaises ou françaises à la même époque.

Il n'en reste pas moins que ces réappropriations sont d'emblée pleines d'ambiguïtés. Chez Klaus Schulze en particulier, les sonorités « cosmiques" de ses albums tranchent avec des titres d'œuvres évoquant la tradition musicale et philosophique allemande du XIX ${ }^{\mathrm{e}}$ siècle, ce que n'a pas manqué de relever la firme Brain qui fit la promotion de son album Timewind avec un slogan qui disait: "Quelle chimie mystérieuse s'opère lorsque la majesté de Wagner rencontre la technologie du $\mathrm{XX}^{\mathrm{e}}$ siècle ? Lorsqu'un millénaire de traditions musicales se mélange avec les sonorités de notre futur électronique $»{ }^{41}$.

Ces lectures contextuelles du rock cosmique ne sont toutefois qu'exceptionnellement confirmées par ses représentants directs. Dans les dizaines d'interviews et articles publiés sur ces groupes entre 1969 et 1973, pas un seul musicien n'évoque la situation de Berlin. On pourrait y voir une sorte d'accomplissement de la théorie du « détournement » évoquée par

41 [Publicité pour Timewind de Klaus Schulze], Melody Maker, 30 août 1975, p. 24. 
Adorno. Ces harmonies, ces rythmes, ces mélodies cosmiques n'auraient eu pour finalité que de détourner les consommateurs des problèmes sociopolitiques. Elles auraient créé - d'une manière presque physique - une sorte d'endormissement de la conscience sociale. Elles fonctionneraient comme un produit létal qui s'ajouterait à la drogue pour permettre d'être ailleurs, comme un moyen de l'avant-garde pour quitter la gravité, fuir le politique, rendre la contestation "stratosphérique » et finalement «mettre en nuage » la contreculture.

En même temps, il est possible aussi de lire le rock cosmique comme une musique de résistance. Résistance évidemment face aux productions musicales convenues d'Europe occidentale ; résistance aux répertoires martiaux dont les sonorités franchissaient le Mur lors des parades et défilés sur la Karl-Marx-Allee et plus fondamentalement résistance contre les formes de pouvoir qui avaient conduit à la séparation de la ville. Leur alternative « cosmique » peut en tout cas être créditée d'un succès essentiel : celui d'avoir fait tomber un mur bien plus ancien que celui de leur ville en rapprochant des traditions «populaires » et « savantes » que l'on pensait immuables. En ce sens, le rock cosmique est prophétie. « Dans ses styles et son organisation économique, [il] est en avance sur le reste de la société parce [qu'il] explore, dans un code donné, tout le champ du possible, plus vite que la réalité matérielle ne peut le faire. [II] fait entendre le monde nouveau qui, peu à peu, deviendra visible... ${ }^{42}$. Il délivre de surcroît le message rassurant d'une possible concorde alors que la Guerre Froide semblait sur le point d'atteindre son point de rupture. 